TRANSACTIONS OF THE

AMERICAN MATHEMATICAL SOCIETY

Volume 353, Number 3, Pages 1169-1182

S $0002-9947(00) 02714-8$

Article electronically published on November 17, 2000

\title{
ALGEBRAIC ISOMORPHISMS OF LIMIT ALGEBRAS
}

\author{
A. P. DONSIG, T. D. HUdSON, AND E. G. KATSOULIS
}

\begin{abstract}
We prove that algebraic isomorphisms between limit algebras are automatically continuous, and consider the consequences of this result. In particular, we give partial solutions to a conjecture and an open problem by Power. As a further consequence, we describe epimorphisms between various classes of limit algebras.
\end{abstract}

In this paper, we study automatic continuity for limit algebras. Automatic continuity involves algebraic conditions on a linear operator from one Banach algebra into another that guarantee the norm continuity of the operator. This is a generalization, via the open mapping theorem, of the uniqueness of norms problem. Recall that a Banach algebra $\mathcal{A}$ is said to have a unique (Banach algebra) topology if any two complete algebra norms on $\mathcal{A}$ are equivalent, so that the norm topology determined by a Banach algebra is unique. Uniqueness of norms, automatic continuity, and related questions have played an important and long-standing role in the theory of Banach algebras [6, 29, 28, 10, 11, 2].

Limit algebras, whose theory has grown rapidly in recent years, are the nonselfadjoint analogues of $\mathrm{UHF}$ and AF $C$-algebras. We first prove that algebraic isomorphisms between limit algebras are automatically continuous (Theorem 1.4). This proof uses the ideal theory of limit algebras as well as key results from the theory of automatic continuity for Banach algebras. Combining this with [23, Theorem 8.3] verifies Power's conjecture that the $C$-envelope of a limit algebra is an invariant for purely algebraic isomorphisms, for limits of finite dimensional nest algebras, and in particular, for all triangular limit algebras (Corollary [1.6]). In [5], the first two authors studied triangular limit algebras in terms of their lattices of ideals. By combining automatic continuity with this work, we show that within the class of algebras generated by their order preserving normalizers (see below for definitions), algebraically isomorphic algebras are isometrically isomorphic (Theorem 2.5]). This shows that the spectrum, or fundamental relation [20, a topological binary relation which provides coordinates for limit algebras and is a useful tool in classifications, is a complete algebraic isomorphism invariant for this class (Corollary 2.6). In recent work, the second two authors studied primitivity for limit algebras [9], showing that a variety of limit algebras are primitive. These results, together with automatic continuity, give descriptions of epimorphisms between various classes of limit algebras, namely lexicographic algebras (Theorem 3.2) and $\mathbb{Z}$-analytic algebras (Theorem 3.3 .

Received by the editors April 6, 1998 and, in revised form, October 7, 1999.

2000 Mathematics Subject Classification. Primary 47D25, 46K50, 46H40.

Research partially supported by an NSF grant.

(C)2000 American Mathematical Society 
We would like to thank the referee for comments about our original "proof" of Theorem 2.5.

Preliminaries. We briefly recall the framework for studying limit algebras; see the monograph [23] for details.

Let $\mathcal{C}$ be an $\mathrm{AF} \mathrm{C}^{*}$-algebra and $X, Y$ subalgebras of $\mathcal{C}$. The normalizer of $Y$ in $X$ is

$N_{Y}(X)=\left\{x \in X \mid x\right.$ is a partial isometry and $x y x^{*}, x^{*} y x \in Y$ for all $\left.y \in Y\right\}$.

A maximal abelian selfadjoint subalgebra (masa) $\mathcal{D}$ of $\mathcal{C}$ is called a canonical masa in $\mathcal{C}$ if there is a nested sequence $\left(C_{k}\right)_{k}$ of finite dimensional $\mathrm{C}^{*}$-subalgebras of $\mathcal{C}$ so that $\mathcal{C}=\overline{\bigcup_{k} C_{k}}$, and if $D_{k}=C_{k} \cap \mathcal{D}$, then $D_{k}$ is a masa in $C_{k}$ satisfying $\mathcal{D}=\overline{\bigcup_{k} D_{k}}$ and $N_{D_{i}}\left(C_{i}\right) \subseteq N_{D_{i+1}}\left(C_{i+1}\right)$. A regular canonical subalgebra is a norm-closed subalgebra of an $\mathrm{AF} \mathrm{C}^{*}$-algebra $\mathcal{C}$ that contains a canonical masa in $\mathcal{~}$. For brevity, we refer to a regular canonical subalgebra as a limit algebra. A norm-closed subalgebra $\mathcal{A}$ of $\mathcal{C}$ is a limit algebra if and only if $\mathcal{A}$ is the direct limit $\underset{\lim }{\longrightarrow}\left(A_{k}, \alpha_{k}\right)$ of a directed system

$$
A_{1} \stackrel{\alpha_{1}}{\longrightarrow} A_{2} \stackrel{\alpha_{2}}{\longrightarrow} A_{3} \stackrel{\alpha_{3}}{\longrightarrow} A_{4} \cdots
$$

where for each $k$,

(i) $A_{k}$ is a subalgebra of the finite dimensional $\mathrm{C}^{*}$-algebra $C_{k}$ containing a masa $D_{k}$ of $C_{k}$,

(ii) $\alpha_{k}$ extends to an injective $*$-homomorphism from $C_{k}$ to $C_{k+1}$, and

(iii) the extension of $\alpha_{k}$ maps $N_{D_{k}}\left(C_{k}\right)$ into $N_{D_{k+1}}\left(C_{k+1}\right)$.

The limit algebra $\mathcal{A}$ is called triangular $A F$ (TAF) if $\mathcal{A} \cap \mathcal{A}^{*}$ equals a canonical masa in $\mathcal{C}$. In this case, each algebra $A_{k}$ in (1) above can be taken to be a subalgebra of some upper triangular matrix algebra. If $\mathcal{A}$ is a TAF algebra and $\mathcal{A}+\mathcal{A}^{*}$ is dense in $\mathcal{C}$, then $\mathcal{A}$ is called strongly maximal triangular. If $\mathcal{A}$ is a strongly maximal TAF algebra, then we can write $\mathcal{A}=\lim \left(A_{k}, \alpha_{k}\right)$, where each $A_{k}$ is a direct sum of $\mathbf{T}_{n}$ 's [16. Lemma 1.1] $\left(\mathbf{T}_{n}\right.$ denotes the $n \times n$ upper triangular matrices with complex entries). If each $A_{k}$ is a single $\mathbf{T}_{n}$ for each $k$, then $\mathcal{A}$ is called strongly maximal triangular in factors. Note that there are strongly maximal triangular subalgebras of $\mathrm{UHF}^{*}$-algebras that are not strongly maximal triangular in factors [16, Example $2.10]$.

\section{Automatic Continuity for algebraic isomorphisms OF LIMIT ALGEBRAS}

To prove that algebraic isomorphisms of limit algebras are continuous, we require Rickart's notion of a separating space. Let $\varphi: \mathcal{A} \rightarrow \mathcal{B}$ be an epimorphism between Banach algebras, and define

$\mathcal{S}(\varphi)=\left\{b \in \mathcal{B} \mid\right.$ there is a sequence $\left(a_{n}\right)_{n} \subseteq \mathcal{A}$ satisfying $a_{n} \rightarrow 0$ and $\left.\varphi\left(a_{n}\right) \rightarrow b\right\}$.

Obviously $\mathcal{S}(\varphi)$ is a closed ideal of $\mathcal{B}$. Moreover, one can easily check that the graph of $\varphi$ is closed if and only if $\mathcal{S}(\varphi)=(0)$. Thus by the closed graph theorem, $\varphi$ is continuous if and only if $\mathcal{S}(\varphi)=(0)$.

The following is an adaptation of [30] Lemma 2.1]. 
Lemma 1.1 (Sinclair). Let $\varphi: \mathcal{A} \rightarrow \mathcal{B}$ be an epimorphism of Banach algebras, and let $\left(b_{n}\right)_{n=1}^{\infty}$ be any sequence in $\mathcal{B}$. Then there exists $N \in \mathbb{N}$ so that for all $n \geq N$,

$$
\overline{b_{1} b_{2} \ldots b_{n} \mathcal{S}(\varphi)}=\overline{b_{1} b_{2} \ldots b_{n+1} \mathcal{S}(\varphi)}
$$

and

$$
\overline{\mathcal{S}(\varphi) b_{n} b_{n-1} \ldots b_{1}}=\overline{\mathcal{S}(\varphi) b_{n+1} b_{n} \ldots b_{1}} .
$$

Proof. Define $a_{n}$ by $\varphi\left(a_{n}\right)=b_{n}$. The first statement follows from 30, Lemma 2.1] with $X=\mathcal{A}, Y=\mathcal{B}$, and $S, T_{n}$, and $R_{n}$ defined as $S x=\varphi(x), T_{n} x=a_{n} x$, and $R_{n} x=b_{n} x$. The second statement follows similarly, but with $S x=\varphi(x)$, $T_{n} x=x a_{n}$, and $R_{n} x=x b_{n}$.

We also require two technical lemmas about ideals in limit algebras. Recall that closed ideals in limit algebras are always inductive [23], that is, if $\mathcal{A}=\overline{\bigcup_{n} A_{n}}$, and $\mathcal{J}$ is an ideal, then $\mathcal{J}=\overline{\bigcup_{n}\left(A_{n} \cap \mathcal{J}\right)}$.

Lemma 1.2. If $\mathcal{J}$ is a nonzero finite dimensional ideal of a limit algebra $\mathcal{A}$, then there exist nonzero projections $p, q$ in $\mathcal{A}$ so that $p \mathcal{A} q=p J q \neq(0)$.

Proof. Let $\lim \left(A_{n}, \alpha_{n}\right)$ be a presentation for $\mathcal{A}$, where each $A_{n}$ is a digraph algebra. Since $\mathcal{J}$ is finite dimensional, for some $k$ there are finitely many matrix units from $A_{k}$ which span J, and moreover, for each $n \geq k$, the image under $\alpha_{n, k}$ of each of these matrix units is a single matrix unit in $A_{n}$. Let $e$ be a matrix unit in $\mathcal{J} \cap A_{k}$, and set $p=e e^{*}, q=e^{*} e$. Clearly, $p J q=\mathbb{C} e \neq(0)$. Since $e$ has only one restriction in $A_{n}$, for each $n \geq k$, both $p$ and $q$ each have only one restriction in each $A_{n}$. It follows that $p A_{n} q=p A_{k} q=p J q$ for each $n \geq k$, so by inductivity, $p \mathcal{A} q=p J q$.

Lemma 1.3. If $\mathcal{J}$ is an infinite dimensional closed ideal in a limit algebra $\mathcal{A}$, then there exists a sequence $\left(p_{n}\right)_{n=1}^{\infty}$ of mutually orthogonal projections in $\mathcal{A}$ so that either $p_{n} \mathcal{J} \neq(0)$ for all $n$ or $\mathcal{J} p_{n} \neq(0)$ for all $n$.

Proof. Let $\lim \left(A_{n}, \alpha_{n}\right)$ be a presentation for $\mathcal{A}$. By relabeling the presentation, if necessary, we can assume $\mathcal{J} \cap A_{1} \neq(0)$. We say $e \in N_{\mathcal{D}}(\mathcal{A})$ has infinitely many restrictions if there is an infinite sequence of pairwise orthogonal projections, $\left(q_{n}\right)$, in $\mathcal{D}$ so that $q_{n} e \neq 0$ for all $n$. Note that, if there is a matrix unit $e$ in $\mathcal{J}$ with infinitely many restrictions, then we can choose the sequence $\left(p_{n}\right)$ from the orthogonal range projections of restrictions of $e$. Let $\Omega$ be the collection of all final projections of matrix units from $\mathcal{J}$ and $\Lambda$ the collection of initial projections. At least one of $\Omega, \Lambda$ must be infinite, for otherwise $\mathcal{J}$ would be finite dimensional.

Assume $\Omega$ is infinite, and let $\mathcal{P}_{n}$ be the set of distinct final projections of all matrix units in $\mathcal{J} \cap A_{n}$. If some $p \in \mathcal{P}_{1}$ had infinitely many restrictions, then a matrix unit in $\mathcal{J} \cap A_{1}$ would have infinitely many restrictions and the conclusion would follow, as noted above. So we may assume that for every $n$, each $p \in \mathcal{P}_{n}$ has only finitely many restrictions. Pick some element $p_{1} \in \mathcal{P}_{1}$. As $p_{1} \in \mathcal{P}_{1} \subseteq \Omega$, $p_{1} \mathcal{J} \neq(0)$. Since $\mathcal{P}_{1}$ is finite and $\Omega$ is infinite, there is some $n>1$ so that $\mathcal{P}_{n}$ contains an element, call it $p_{2}$, which is not a restriction of some $p \in \mathcal{P}_{1}$. Since it is not a restriction of any element of $\mathcal{P}_{1}, p_{2}$ may be chosen orthogonal to each element of $\mathcal{P}_{1}$, and in particular, to $p_{1}$. Again, $p_{2} \mathcal{J} \neq(0)$. Next, there is some $m>n$ so that $\mathcal{P}_{m}$ contains an element, call it $p_{3}$, which is not a restriction of some $p \in \mathcal{P}_{n}$. Continuing in this way, we can construct the required sequence $\left(p_{n}\right)_{n=1}^{\infty}$ with $p_{n} \mathcal{J} \neq(0)$. 
With the obvious modifications, the same argument works if $\Lambda$ is infinite.

We can now prove our main result.

Theorem 1.4. If $\varphi: \mathcal{A} \rightarrow \mathcal{B}$ is an algebraic isomorphism between limit algebras $\mathcal{A}$ and $\mathcal{B}$, then $\varphi$ is continuous.

Proof. It suffices to prove that $\mathcal{S}(\varphi)=(0)$. Assume that $\mathcal{S}(\varphi)$ is nonzero and finite dimensional. By Lemma 1.2, there are nonzero projections $p, q \in \mathcal{B}$ so that $p \mathcal{B} q=p \mathcal{S}(\varphi) q \neq(0)$. In particular, $p \mathcal{B} q$ is finite dimensional. Let $e, f \in \mathcal{A}$ satisfy $\varphi(e)=p$ and $\varphi(f)=q$. Clearly $e, f$ are idempotents in $\mathcal{A}$, and $e \mathcal{A} f$ is finite dimensional since $p \mathcal{B} q$ is.

Now let $b \in \mathcal{S}(\varphi)$ so that $p b q \neq 0$. Then there is a sequence $\left(a_{n}\right)_{n}$ in $\mathcal{A}$ so that $a_{n} \rightarrow 0$ and $\varphi\left(a_{n}\right) \rightarrow b$. Hence

$$
\varphi\left(e a_{n} f\right)=p \varphi\left(a_{n}\right) q \longrightarrow p b q \neq 0 .
$$

But since $e a_{n} f \rightarrow 0$, this shows that the map $\left.\varphi\right|_{e \mathcal{A} f}$ is discontinuous. But $\operatorname{dim}(e \mathcal{A} f)$ $<\infty$, a contradiction.

Hence if $\mathcal{S}(\varphi)$ is nonzero, it must be infinite dimensional. Applying Lemma 1.3 to the ideal $\mathcal{S}(\varphi)$, we obtain a sequence $\left(p_{n}\right)_{n=1}^{\infty}$ of mutually orthogonal projections in $\mathcal{B}$ so that either $p_{n} \mathcal{S}(\varphi) \neq(0)$ for each $n$ or $\mathcal{S}(\varphi) p_{n} \neq(0)$ for each $n$. Set

$$
b_{n}=\sum_{k=n}^{\infty} \frac{p_{k}}{2^{k}}
$$

so that

$$
b_{1} b_{2} \ldots b_{n}=b_{n} b_{n-1} \ldots b_{1}=\sum_{k=n}^{\infty} \frac{p_{k}}{2^{n k}} .
$$

If $p_{n} \mathcal{S}(\varphi) \neq(0)$ for each $n$, then

$$
\overline{b_{1} b_{2} \ldots b_{n} \mathcal{S}(\varphi)} \supsetneq \overline{b_{1} b_{2} \ldots b_{n+1} \mathcal{S}(\varphi)} \text {. }
$$

If $\mathcal{S}(\varphi) p_{n}$ is nonzero for each $n$, then

$$
\overline{\mathcal{S}(\varphi) b_{n} b_{n-1} \ldots b_{1}} \supsetneq \overline{\mathcal{S}(\varphi) b_{n+1} b_{n} \ldots b_{1}} .
$$

In either case, we have contradicted Theorem 1.1. It follows that if $\varphi$ is an algebraic isomorphism, then $\mathcal{S}(\varphi)=(0)$, i.e., $\varphi$ is continuous.

Remark. A similar result for triangular subalgebras of groupoid $\mathrm{C}^{*}$-algebras was previously announced and circulated in preprint form [27, Theorem 6.4]. Unfortunately, the proof given there is incomplete. In any case, our techniques are quite different and Theorem 1.4 applies to non-triangular algebras, albeit subalgebras of AF $\mathrm{C}^{*}$-algebras.

The above proof, with only trivial changes, also establishes the following result.

Corollary 1.5. If $\mathcal{A}$ is a Banach algebra and $\mathcal{B}$ a limit algebra with no finite dimensional ideals, then any epimorphism from $\mathcal{A}$ onto $\mathcal{B}$ is continuous.

Power has shown that the $C^{*}$-envelope of certain limit algebras is a Banach algebra invariant [23, Theorem 8.3]. Note that Theorem 8.3 is not valid in the generality stated, as it depends on Lemma 8.1, which is false [26]; thus, Theorem 8.3 must be restricted to inductive limits of finite dimensional nest algebras. However, 
this includes all strongly maximal triangular limit algebras, so the theorem still applies to a wide range of algebras.

Power has suggested that the $C^{*}$-envelope is an invariant for purely algebraic isomorphisms of limit algebras [23, Chapter 8 Notes]. Power's work and Theorem 1.4 directly imply this conjecture for a variety of limit algebras.

Corollary 1.6. If $\mathcal{A}$ and $\mathcal{B}$ are algebraically isomorphic limit algebras, both inductive limits of finite dimensional nest algebras, then $C^{*}(\mathcal{A})$ and $C^{*}(\mathcal{B})$ are isomorphic as $\mathrm{C}^{*}$-algebras.

Moreover, Theorem 1.4 implies that to show the $C^{*}$-envelope is an algebraic invariant for all limit algebras, it is enough to show that it is a Banach algebra invariant.

\section{Algebraic isomorphisms Between order PRESERVIng TAF ALgebras}

Power has conjectured that algebraic isomorphism may be equivalent to isometric isomorphism for subalgebras of $\mathrm{AF}^{*}$-algebras containing a regular canonical masa [22, p. 95]. In this section we combine Theorem 1.4 with results from [5] to prove this conjecture for strongly maximal TAF algebras generated by their order preserving normalizers. That is, two algebras in this family are algebraically isomorphic if and only if they are isometrically isomorphic.

This family includes a range of fundamental examples, such as the standard, refinement, and alternation limit algebras [1 7, 15 17], the lexicographic algebras [14, 24, 25], and the $\mathbb{Z}$-analytic algebras [16, 18, as well as non-analytic algebras [8, 31]. We include the necessary definitions below, but refer the reader to [3, 5] for further details on order preservation and TAF algebras generated by their order preserving normalizers.

Fix a TAF algebra $\mathcal{A}$, and let $\mathcal{P}(\mathcal{A})$ denote the collection of all diagonal projections of $\mathcal{A}$. The diagonal order on $\mathcal{P}(\mathcal{A})$, denoted " $\preceq$ ", is a partial order given by

$$
e \preceq f \Longleftrightarrow \text { there exists } v \in N_{\mathcal{D}}(\mathcal{A}) \text { with } v v^{*}=e, v^{*} v=f .
$$

Each $v \in N_{\mathcal{D}}(\mathcal{A})$ induces a partial homeomorphism on $\mathcal{P}(\mathcal{A})$, which has domain $\left\{x \in \mathcal{P}(\mathcal{A}) \mid x \leq v v^{*}\right\}$ and range $\left\{x \in \mathcal{P}(\mathcal{A}) \mid x \leq v^{*} v\right\}$, given by $x \longmapsto v^{*} x v$. We say that $v$ is order preserving if this map preserves the diagonal order restricted to its domain and range. Define the order preserving normalizer of $\mathcal{A}$ to be

$$
N_{\mathcal{D}}^{\text {ord }}(\mathcal{A})=\left\{v \in N_{\mathcal{D}}(\mathcal{A}) \mid w \text { is order preserving }\right\},
$$

and let $\mathcal{A}^{\text {ord }}$ be the subalgebra of $\mathcal{A}$ generated by $N_{\mathcal{D}}^{\text {ord }}(\mathcal{A})$. Note that a sum of elements of $N_{\mathcal{D}}^{\text {ord }}(\mathcal{A})$ may not be in $N_{\mathcal{D}}^{\text {ord }}(\mathcal{A})$, even if the sum is in $N_{\mathfrak{D}}(\mathfrak{A})$. Also, note that if $v \in N_{\mathcal{D}}^{\text {ord }}(\mathcal{A})$ and $p \in \mathcal{D}$ is a projection, then $p v$ and $v p$ are in $N_{\mathcal{D}}^{\text {ord }}(\mathcal{A})$.

An embedding $\alpha: A \rightarrow B$ between triangular algebras is called locally order preserving if $\alpha(v)$ is an order preserving element of $B$ for every matrix unit $v \in A$. For example, standard embeddings, refinement embeddings, embeddings induced by ordered Bratteli diagrams (see [23, Chapter 6]) and the block standard embeddings of [8] are all locally order preserving. This is easy to verify using the following characterization.

Theorem 2.1 ([3, Theorem 18]). A TAF algebra $\mathcal{A}$ is generated by its order preserving normalizer, if and only if, it has a presentation $\lim \left(A_{i}, \alpha_{i}\right)$ with $\alpha_{j} \circ \cdots \circ \alpha_{i}$ locally order preserving for all $i \leq j$. 
Lemma 2.2. Suppose $\mathcal{A}$ is a strongly maximal TAF algebra with diagonal $\mathcal{D}$. If $e, f \in N_{\mathcal{D}}(\mathcal{A})$ and $e+f \in N_{\mathcal{D}}^{\text {ord }}(\mathcal{A})$, then neither $e$ nor $f$ is in the ideal generated by the other.

Proof. Let $\lim \left(A_{i}, \alpha_{i}\right)$ be a presentation for $\mathcal{A}$. By [15, Theorem 3.6] or [20. Lemma 6.3], $e=c x$ and $f=d y$ where $c, d$ are partial isometries in $\mathcal{D}$ and $x, y$ are sums of matrix units in some $A_{l}$ with the same initial and final projections as $e$ and $f$, respectively. Then $e+f \in N_{\mathcal{D}}^{\text {ord }}(\mathcal{A})$ implies $x+y \in N_{\mathcal{D}}^{\text {ord }}(\mathcal{A})$ and $x$ and $y$ generate the same ideals as $e$ and $f$, respectively. Thus, we may assume that $e$ and $f$ are sums of matrix units in $A_{l}$, for some $l$.

For the sake of contradiction, suppose that $f$ is in the ideal generated by $e$. Let $J_{n}$ be the ideal of $A_{n}$ generated by $e$. Although $f$ need not be in $J_{l}$ (see, for example [8, Example 1.6]), there is some $k \geq l$ with $f \in J_{k}$ by [8] Proposition 1.7]. In particular, there are individual matrix units of $A_{k}, e_{1}$ in the sum for $e$ in $A_{k}$ and $f_{1}$ in the sum for $f$ in $A_{k}$, so that $a e_{1} b=f_{1}$ for matrix units $a, b \in A_{k}$. Since $N_{\mathcal{D}}^{\text {ord }}(\mathcal{A})$ is closed under multiplication by diagonal projections, $e+f \in N_{\mathcal{D}}^{\text {ord }}(\mathcal{A})$ implies $e_{1}+f_{1} \in N_{\mathcal{D}}^{\text {ord }}(\mathcal{A})$. By the definition of diagonal order, $a^{*} a \preceq a a^{*}$ and similarly for $b$. However, conjugation by $e_{1}+f_{1}$ sends $b b^{*}$ to $a^{*} a$ and $b^{*} b$ to $a a^{*}$, reversing the order. This shows $e_{1}+f_{1} \notin N_{\mathcal{D}}^{\text {ord }}(\mathcal{A})$, the promised contradiction.

We will need the following result.

Theorem 2.3 ([5, Corollary 18]). Let $\mathcal{A}$ and $\mathcal{B}$ be strongly maximal TAF algebras generated by their order preserving normalizers, and assume that $\phi: \operatorname{Id}(\mathcal{A}) \rightarrow \operatorname{Id}(\mathcal{B})$ is an isomorphism of the lattices of (closed, two-sided) ideals.

Then there is a bijective isometry $\eta: \mathcal{A} \rightarrow \mathcal{B}$ and closed triangular subalgebras $\mathcal{E}$ and $\mathcal{F}$ of $\mathcal{A}$ with $\mathcal{E}+\mathcal{F}=\mathcal{A}$ and $\mathcal{E} \cap \mathcal{F}=\mathcal{A} \cap \mathcal{A}^{*}$, so that $\eta$ is an algebra isomorphism on $\mathcal{E}$ and an anti-isomorphism on $\mathcal{F}$. Moreover, the induced action of $\eta$ on the lattices of ideals agrees with $\phi$.

The spectrum, or fundamental relation, of a limit algebra was introduced by Power 21] and is a complete isometric isomorphism invariant for TAF algebras. For a limit algebra $\mathcal{A}$, let $H$ denote the maximal ideal space of the canonical masa $\mathcal{D} \subseteq \mathcal{A}$. The spectrum of $\mathcal{A}$ is the topological binary relation $\mathrm{R}(\mathcal{A})$ on $H$ such that $(x, y) \in \mathrm{R}(\mathcal{A})$ if and only if there is an element $v$ of $N_{\mathcal{D}}(\mathcal{A})$ so that $y(d)=x\left(v d v^{*}\right)$ for all $d$ in $\mathcal{D}$. In this case we say that $v$ relates $x$ and $y$. If $v \in N_{\mathcal{D}}(\mathcal{A})$, define $G(v)$ to be the graph of $v$ in $\mathrm{R}(\mathcal{A})$, that is,

$$
G(v)=\{(x, y) \in \mathrm{R}(\mathcal{A}) \mid v \text { relates } x \text { and } y\} .
$$

Consider the topology on $\mathrm{R}(\mathcal{A})$ that has $\left\{G(v) \mid v \in N_{\mathcal{D}}(\mathcal{A})\right\}$ as a base of open sets. The sets $G(v)$ are then clopen and compact, and so $\mathrm{R}(\mathcal{A})$ is a totally disconnected, locally compact Hausdorff space. Let $\lim \left(A_{i}, \alpha_{i}\right)$ be a presentation for $\mathcal{A}$. If $v \in$ $N_{\mathcal{D}}(\mathcal{A})$, then by [15, Theorem 3.6] or [20, Lemma 6.3], $v=c w$ where $c$ is a partial isometry in $\mathcal{C}$ and $w$ is a sum of matrix units in some $A_{l}$. Hence, for all $i \geq l$, conjugation by $v$ carries diagonal matrix units in $A_{i}$ to diagonal matrix units in $A_{i}$ or zero. Moreover, it follows that the topology on $\mathrm{R}(\mathcal{A})$ is completely determined by those $G(v)$ for which $v$ is a matrix unit.

The maximal ideal space of $\mathcal{D}$ (and hence the spectrum) can also be described in terms of decreasing sequences of diagonal projections. We outline this description. Again, let $\underset{\lim }{\longrightarrow}\left(A_{i}, \alpha_{i}\right)$ be a presentation for $\mathcal{A}$. Given a maximal ideal $x$ of $\mathcal{D}$, we 
associate a sequence of diagonal matrix units $\left(p_{i}\right)$ where each $p_{i} \in A_{i}$ is the unique diagonal matrix unit with $x\left(p_{i}\right) \neq 0$. Conversely, if $\left(p_{i}\right)$ is a decreasing sequence of matrix units, where each $p_{i}$ is a minimal diagonal projection in $A_{i}$, then the associated maximal ideal $x$ is defined as the direct limit of the linear multiplicative maps $x_{i}: A_{i} \cap A_{i}^{*} \rightarrow \mathbb{C}$ with $x_{i}\left(p_{i}\right)=1$ and $x_{i}(c)=0$ for all other diagonal matrix units $c$.

More generally, let $\left(p_{i}\right)$ be a decreasing sequence of diagonal projections satisfying the following:

For each projection $d \in \mathcal{D}$, there is $j \in \mathbb{N}$ with either

$$
d p_{i}=0, \forall i \geq j \text {, or } d p_{i}=p_{i}, \forall i \geq j .
$$

For such a sequence of projections, one can still associate a unique maximal ideal, namely $\bigcap_{i} G\left(p_{i}\right)$. To see that this is nonempty, observe that it is a decreasing sequence of nonempty compact sets. To see that it is a singleton, suppose $x, y$ are distinct maximal ideals in $\bigcap_{i} G\left(p_{i}\right)$. As the maximal ideal space is Hausdorff and totally disconnected, there is closed and open neighbourhood of $x$ that does not contain $y$, that is, a projection $p$ with $x \in G(p)$ and $y \notin G(p)$. By condition $(*)$, there is some $j$ so that $G\left(p_{i}\right) \subset G(p)$ for all $i \geq j$ or $G\left(p_{i}\right) \cap G(p)=\emptyset$ for all $i \geq j$. In the first case, we contradict $y \in \cap_{i} G\left(p_{i}\right)$ and in the second we contradict $x \in \cap_{i} G\left(p_{i}\right)$. Thus, $\bigcap_{i} G\left(p_{i}\right)$ is the unique maximal ideal not containing any of the $p_{i}$.

We can describe the spectrum in terms of decreasing sequences of projections.

Lemma 2.4. Suppose $\left(p_{i}\right)$ and $\left(q_{i}\right)$ are decreasing sequences of projections in a strongly maximal TAF algebra $\mathcal{A}$ satisfying Condition $(*)$. Let $x$ and $y$ be the maximal ideals of the diagonal $\mathcal{D}$ given by $\{x\}=\bigcap_{i} G\left(p_{i}\right)$ and $\{y\}=\bigcap_{i} G\left(q_{i}\right)$ and suppose $v \in N_{\mathcal{D}}(\mathcal{A})$. If $(x, y) \notin G(v)$, then there is $j \in \mathbb{N}$ so that $p_{i} v q_{i}=0$ for all $i \geq j$.

Proof. Applying Condition (*) with $d=v v^{*}$, either $p_{j} v=0$ for some $j$ or $p_{i}$ is a subprojection of $v v^{*}$ for all $i$. If $p_{j} v=0$, then $p_{i} v q_{i}=0$, for all $i \geq j$. Thus we may assume that $p_{i}$ is a subprojection of $v v^{*}$ for all $i$. Similarly, we may assume $q_{i}$ is a subprojection of $v^{*} v$ for all $i$.

By hypothesis, there is some projection $d \in \mathcal{D}$ with $y(d) \neq x\left(v d v^{*}\right)$. Suppose first that $y(d)=1$ and $x\left(v d v^{*}\right)=0$. As $y(d)=1$, there is $k \in \mathbb{N}$ with $q_{i} d=q_{i}$ for all $i \geq k$. As $x\left(v d v^{*}\right)=0$, we have $x\left(v q_{k} v^{*}\right)=0$. Since $x\left(p_{i}\right)=1$ for all $i$, there is $j \geq k$ with $p_{j}\left(v q_{k} v^{*}\right)=0$ and hence $p_{j} v q_{j} v^{*}=0$. As $q_{j}$ is a subprojection of $v^{*} v$, it follows that $p_{j} v q_{j}=0$. Hence $p_{i} v q_{i}=0$ for all $i \geq j$.

On the other hand, if $y(d)=0$ and $x\left(v d v^{*}\right)=1$, then there is $k \in \mathbb{N}$ with $p_{i} v d v^{*}=p_{i}$ for all $i \geq k$. It follows that $v^{*} p_{k} v$ is a subprojection of $d$ and so $y\left(v^{*} p_{k} v\right)=0$. As $y\left(q_{i}\right)=1$ for all $i$, there is $j \geq k$ with $\left(v^{*} p_{k} v\right) q_{j}=0$ and so $v^{*} p_{j} v q_{j}=0$. Arguing as before, we have $p_{j} v q_{j}=0$.

Finally, we claim that if $\mathcal{J}$ is a maximal ideal of a limit algebra with diagonal $\mathcal{D}$, then $\mathcal{J} \cap \mathcal{D}$ is a maximal ideal in $\mathcal{D}$. To see the claim observe that $1 \in \mathcal{D}$ so there is some maximal ideal $\mathcal{J}$ of $\mathcal{D}$ with $\mathcal{J} \cap \mathcal{D} \subseteq \mathcal{J}$. If $\mathcal{K}$ is the ideal generated by $\mathcal{J}$ and all off-diagonal matrix units of $\mathcal{A}$ (in some presentation), then $\mathcal{K}$ is a proper ideal of $\mathcal{A}$ containing $\mathcal{J}$. By the maximality of $\mathcal{J}, \mathcal{J}=\mathcal{K}$. Thus $\mathcal{J} \cap \mathcal{D}=\mathcal{K} \cap \mathcal{D}=\mathcal{J}$, which proves the claim. By this claim, if $(x, y) \in R(A)$, we may regard $x$ and $y$ as maximal ideals of $\mathcal{D}$ or $\mathcal{A}$, as convenient 
The key provided by automatic continuity is that an algebraic isomorphism between limit algebras induces a lattice isomorphism between their lattices of closed ideals. Theorem 2.3 then yields a bijective isometry between the algebras, which is then deduced to be an isomorphism.

Theorem 2.5. Suppose $\mathcal{A}$ and $\mathcal{B}$ are strongly maximal TAF algebras generated by their order preserving normalizers. Then $\mathcal{A}$ and $\mathcal{B}$ are algebraically isomorphic if and only if they are isometrically isomorphic.

Proof. One direction is trivial; to prove the other, suppose that $\phi: \mathcal{A} \rightarrow \mathcal{B}$ is an algebraic isomorphism.

Theorem 1.4 shows that an algebra isomorphism is automatically continuous, and so $\phi$ maps closed ideals to closed ideals. Thus it induces a lattice isomorphism which, by Theorem 2.3 is induced by a bijective isometry $\eta: \mathcal{A} \rightarrow \mathcal{B}$ with the properties described in that theorem. We will show that $\mathcal{F}=\mathcal{A} \cap \mathcal{A}^{*}$ and so $\mathcal{E}=\mathcal{A}$, proving the theorem.

Let $\mathcal{C}=\mathcal{A} \cap \mathcal{A}^{*}, \mathcal{D}=\mathcal{B} \cap \mathcal{B}^{*}$ and let $P$ be the conditional expectation from $\mathcal{B}$ to $\mathcal{D}$.

Claim 1. $\eta(x)=(P \circ \phi)(x)$ for all $x \in \mathcal{C}$.

It is enough to prove the claim for $x$ a projection in $\mathcal{C}$. Observe that $\phi$ and $\eta$ induce the same isomorphism from the maximal ideals of $\mathcal{A}$ to the maximal ideals of $\mathcal{B}$. Clearly $\phi(x)$ and $\eta(x)$ belong to the same maximal ideals of $\mathcal{B}$. Since any multiplicative linear form coincides on $\phi(x)$ and $(P \circ \phi)(x)$, we conclude that $(P \circ \phi)(x)$ and $\eta(x)$ belong to the same maximal ideals of $\mathcal{B}$, and hence of $\mathcal{D}$. We claim that $P$ is multiplicative and hence $P(\phi(x))$ is an idempotent in the abelian $\mathrm{C}^{*}$-algebra $\mathcal{D}$ and so is a projection. Viewing $(P \circ \phi)(x)$ and $\eta(x)$ as characteristic functions over the maximal ideal space, Claim 1 is proved.

To see the claim, note that $\mathcal{B}$ decomposes as $\mathcal{D} \oplus \mathcal{J}$ where $\mathcal{J}$ is the ideal generated by the off-diagonal matrix units in a presentation of $\mathcal{B}$. Since the kernel of $P$ is $\mathcal{J}$, a short calculation establishes that $P$ is multiplicative.

As $\mathcal{F} \neq \mathcal{C}$ and $N_{\mathcal{C}}^{\text {ord }}(\mathcal{A})$ generates $\mathcal{A}$, we can choose some $x \in N_{\mathrm{C}}^{\mathrm{ord}}(\mathcal{A}) \cap \mathcal{F}$ with $Q(x)=0$, where $Q: \mathcal{A} \rightarrow \mathcal{C}$ is the conditional expectation. If $\mathcal{A}=\underline{\lim }\left(A_{i}, \alpha_{i}\right)$, then we may regard $\mathcal{A}$ as the closed union of the $A_{i}$. By [15, Theorem 3.6] or [20. Lemma 6.3], $x=c w$ where $c \in \mathcal{C}$ and $w$ is a sum of matrix units in some $A_{l}$. Since $\mathcal{F}$ and $\operatorname{ker} Q$ are $\mathcal{C}$-bimodules, we have that $w \in \mathcal{F}$ and $Q(w)=0$. Thus, we may assume that $x$ is a sum of matrix units in $A_{i}$ for all $i$ sufficently large.

Since $\eta$ is an isometric algebra anti-isomorphism on $\mathcal{F}$, [20, Lemma 6.4] implies that $\eta(x) \in N_{\mathcal{D}}(\mathcal{B})$. Notice that the initial projection of $\eta(x)$ is $\eta\left(x x^{*}\right)$ and the final projection is $\eta\left(x^{*} x\right)$. Indeed, since $x\left(x^{*} x\right)=x$, we have $\eta\left(x^{*} x\right) \eta(x)=\eta(x)$ and so $\eta(x) \eta(x)^{*} \leq \eta\left(x^{*} x\right)$. On the other hand, for any projection $p \in \mathcal{D}$ with $p \eta(x)=\eta(x), x \eta^{-1}(p)=x$ and so $\eta^{-1}(p) \geq x^{*} x$. This implies that $p \geq \eta\left(x^{*} x\right)$, as $\eta(\mathcal{C})=\mathcal{D}$ and so $\eta$ and $\eta^{-1}$ are positive. Letting $p=\eta(x) \eta(x)^{*}$ gives the reverse inclusion. The argument that $\eta(x)^{*} \eta(x)=\eta\left(x x^{*}\right)$ is similar.

Let $M_{1}$ and $M_{2}$ be maximal ideals of $\mathcal{C}$ with $\left(M_{1}, M_{2}\right)$ in $G(x)$. We identify $M_{1}$ and $M_{2}$ with maximal ideals of $\mathcal{A}$. Then $N_{1}=\eta\left(M_{1}\right)$ and $N_{2}=\eta\left(M_{2}\right)$ are maximal ideals of $\mathcal{B}$, which we can identify with maximal ideals of $\mathcal{D}$. Moreover, since $\eta(x)$ is a normalizing partial isometry and $\eta$ is anti-multiplicative on $x, x^{*} x$, and $x x^{*}$, $\left(N_{2}, N_{1}\right)$ is $G(\eta(x))$. Note that $N_{1} \neq N_{2}$ as otherwise $M_{1}=M_{2}$ and then $G(x)$ intersects the diagonal of the spectrum, contradicting $Q(x)=0$. 
Since $\mathcal{B}$ is triangular, the spectrum of $\mathcal{B}$ is anti-symmetric and so $\left(N_{1}, N_{2}\right)$ is not in the spectrum of $\mathcal{B}$. We will use this to obtain a contradiction.

Let $\left(p_{i}\right)_{i}$ and $\left(q_{i}\right)_{i}$ be decreasing sequences of projections associated to $M_{1}$ and $M_{2}$ respectively, as discussed before Theorem [2.3. In particular $\left(p_{i}\right)$ and $\left(q_{i}\right)$ satisfy condition $(*)$. Clearly, the sequences $\left(\eta\left(p_{i}\right)\right)_{i}$ and $\left(\eta\left(q_{i}\right)\right)_{i}$ satisfy the condition $(*)$ and are associated to $N_{1}$ and $N_{2}$. Let

$$
x_{i}=p_{i} x q_{i} \quad \text { and } \quad \alpha_{i}=\eta\left(p_{i}\right) \phi(x) \eta\left(q_{i}\right) .
$$

Claim 2. $\lim _{i \rightarrow \infty} \alpha_{i}=0$.

Since $\left(N_{1}, N_{2}\right) \notin \mathrm{R}(\mathcal{B})$, for each $v \in N_{\mathcal{D}}(\mathcal{B})$, Lemma 2.4 shows that there is some $j$ so that $\eta\left(p_{i}\right) v \eta\left(q_{i}\right)=0$ for all $i \geq j$. Given $\epsilon>0$, we may approximate $\phi(x)$ to within $\epsilon$ by some element $z$ from a finite-dimensional algebra $B_{n} \subset \mathcal{B}$. Since $B_{n}$ has finitely many matrix units, there is some $j$ so that $\eta\left(p_{i}\right) y \eta\left(q_{i}\right)=0$ for all matrix units $y$ in $B_{n}$ and all $i \geq j$. Thus $\eta\left(p_{i}\right) z \eta\left(q_{i}\right)=0$ and so $\alpha_{i}=\eta\left(p_{i}\right) \phi(x) \eta\left(q_{i}\right)$ has norm at most $\epsilon$ for all $i \geq j$. This proves Claim 2 .

Claim 2 and the continuity of $\phi^{-1}$ imply that the sequence $\phi^{-1}\left(\alpha_{i}\right)$ also converges to zero. Note that

$$
\phi^{-1}\left(\alpha_{i}\right)=\phi^{-1}\left(\eta\left(p_{i}\right)\right) x \phi^{-1}\left(\eta\left(q_{i}\right)\right)=\phi^{-1}\left(P \circ \phi\left(p_{i}\right)\right) x \phi^{-1}\left(P \circ \phi\left(q_{i}\right)\right) .
$$

We have that

$$
\phi^{-1}\left(\alpha_{i}\right)=x_{i}+x_{i} z_{i}+y_{i} x_{i}+y_{i} x z_{i}
$$

where $\phi^{-1}\left(P\left(\phi\left(p_{i}\right)\right)\right)=p_{i}+y_{i}$ and $\phi^{-1}\left(P\left(\phi\left(q_{i}\right)\right)\right)=q_{i}+z_{i}$, for some elements $y_{i}$ and $z_{i}$ in the zero-diagonal ideal of $\mathcal{A}$. To see that $y_{i}$ and $z_{i}$ are in the zero-diagonal ideal of $\mathcal{A}$, observe that this ideal is the intersection of the maximal ideals of $\mathcal{A}$ and similarly for the zero-diagonal ideal of $\mathcal{B}$. Since $\phi^{-1}$ maps maximal ideals to maximal ideals, it sends the zero-diagonal ideal of $\mathcal{B}$ to the zero-diagonal ideal of $\mathcal{A}$. As $y_{i}$ is $\phi^{-1}$ applied to the zero-diagonal element $(P-1)\left(\phi\left(p_{i}\right)\right)$, we have $y_{i}$ zero-diagonal. Similar arguments apply to $z_{i}$.

Choose $i$ sufficiently large that $\phi^{-1}\left(\alpha_{i}\right)$ has norm less than $1 / 4$ and $x$ is a sum of matrix units in $A_{i}$. For some $n \geq i$, we can find $z^{\prime} \in A_{n}$ with

$$
\left\|z^{\prime}-z_{i}\right\| \leq \min \left\{\frac{1}{16\left\|x_{i}\right\|}, \frac{1}{16\left\|y_{i} x\right\|}\right\}
$$

and then find $y^{\prime} \in A_{n}$ with

$$
\left\|y^{\prime}-y_{i}\right\| \leq \min \left\{\frac{1}{16\left\|x_{i}\right\|}, \frac{1}{16\left\|x z^{\prime}\right\|}\right\} .
$$

Notice that $y_{i}$ and $z_{i}$ are in the zero-diagonal ideal of $\mathcal{A}$ and so we may choose $y^{\prime}$ and $z^{\prime}$ to be in the zero-diagonal ideal of $A_{n}$. Routine calculations show that

$$
\left\|\left(x_{i} z_{i}+y_{i} x_{i}+y_{i} x z_{i}\right)-\left(x_{i} z^{\prime}+y^{\prime} x_{i}+y^{\prime} x z^{\prime}\right)\right\| \leq \frac{1}{4} .
$$

As $\left\|\left(-x_{i}\right)-\left(x_{i} z_{i}+y_{i} x_{i}+y_{i} x z_{i}\right)\right\|=\left\|\phi^{-1}\left(\alpha_{i}\right)\right\| \leq 1 / 4$, we have

$$
\left\|\left(-x_{i}\right)-\left(x_{i} z^{\prime}+y^{\prime} x_{i}+y^{\prime} x z^{\prime}\right)\right\| \leq \frac{1}{2} .
$$

On the other hand, observe that $x$ and hence $x_{i}$ are sums of matrix units in $A_{n}$, which is a direct sum of upper-triangular matrix algebras. Let $e$ be a matrix unit in the sum for $x_{i}$. By Lemma 2.2 as $x, x_{i} \in N_{\mathcal{D}}^{\text {ord }}(\mathcal{A}), e$ is not in the ideals generated by $x-e$ and $x_{i}-e$. Since $z^{\prime}$ and $y^{\prime}$ are linear combinations of strictly upper 
triangular matrix units, the linear combinations of matrix units $e z^{\prime}, y^{\prime} e$ and $y^{\prime} e z^{\prime}$ do not include non-zero multiples of the matrix unit $e$. As $x_{i} z^{\prime}=\left(x_{i}-e\right) z^{\prime}+e z^{\prime}$, $x_{i} z^{\prime}$ does not include a non-zero multiple of $e$, and similarly for $y^{\prime} x_{i}$ and $y^{\prime} x z^{\prime}$. Hence, in the expression $\left(-x_{i}\right)-\left(x_{i} z^{\prime}+y^{\prime} x_{i}+y^{\prime} x z^{\prime}\right) \in A_{n}$, only the first term contains a non-zero multiple of $e$. As the coefficient of $e$ in $x_{i}$ is 1 , we have

$$
\left\|\left(-x_{i}\right)-\left(x_{i} z^{\prime}+y^{\prime} x_{i}+y^{\prime} x z^{\prime}\right)\right\| \geq\|e\|=1 .
$$

Hence no such $x$ in $N_{\mathfrak{C}}^{\text {ord }}(\mathcal{A}) \cap(\mathcal{F} \backslash \mathcal{C})$ exists and so $\mathcal{F}=\mathcal{C}$.

For triangular limit algebras, the spectrum is an isometric isomorphism invariant [21, Theorem 3] and it has been asked if this extends to algebraic isomorphism: 21] and [23, Problem 7.8]. Theorem 2.5 combined with the cited result yields a partial solution to this problem.

Corollary 2.6. The spectrum or fundamental relation $\mathrm{R}(\mathcal{A})$ is a complete algebraic isomorphism invariant for strongly maximal TAF algebras $\mathcal{A}$ generated by their order preserving normalizers.

In addition to this result, automatic continuity can be used to provide further evidence for a positive solution to the problem. That is, we can show that certain not isometrically isomorphic TAF algebras are not algebraically isomorphic, even though some of these algebras are not generated by their order preserving normalizers.

Example 2.7. Let $A_{n}=\mathbf{T}_{2^{n}}$ and define the multiplicity 2 refinement embedding $\rho_{k}: A_{k} \rightarrow A_{k+1}$ by

$$
\rho_{k}\left(\left[a_{i j}\right]\right)=\left[a_{i j} I_{2}\right],
$$

where $I_{2}$ is the $2 \times 2$ identity matrix. Now define the elementary twist embedding $\tau_{k}: A_{k} \rightarrow A_{k+1}$ by

$$
\tau_{k}=\left(\operatorname{Ad} U_{2^{n+1}}\right) \circ \rho_{k}
$$

where $U_{2^{n+1}}$ is the permutation unitary in $\mathbf{M}_{2^{n+1}}$ which interchanges the last two minimal diagonal projections of $A_{n+1}$. Let $\mathcal{A}=\lim \left(A_{n}, \rho_{n}\right)$ and $\mathcal{B}=\lim \left(A_{n}, \tau_{n}\right)$.

The TAF algebras $\mathcal{A}$ and $\mathcal{B}$ are called the $2^{\infty}$ refinement and refinement with twist algebras, respectively.

One can tell that there is no isometric isomorphism between $\mathcal{A}$ and $\mathcal{B}$ by examining their spectrums [21]. But a more delicate question is whether there is an algebraic isomorphism between $\mathcal{A}$ and $\mathcal{B}$. If there were such an isomorphism $\phi: \mathcal{A} \rightarrow \mathcal{B}$, Theorem 1.4 shows that $\phi$ would be continuous. Hence, since $\phi$ then maps closed ideals of $\mathcal{A}$ to closed ideals of $\mathcal{B}, \phi$ induces a lattice isomorphism between the lattices of closed ideals of $\mathcal{A}$ and $\mathcal{B}$. But the sentence before Corollary 28 in [5] shows there exists no such lattice isomorphism between the lattices of ideals of the $2^{\infty}$ refinement and the refinement with twist, and hence there is no algebraic isomorphism between $\mathcal{A}$ and $\mathcal{B}$.

Using an argument similar to the proof of [5. Corollary 28], one can show that there are no algebraic isomorphisms between pairs of more general "twist" type algebras, as considered in [5] Section 5]. 


\section{EPIMORPHISMS}

The class of TAF algebras generated by their order preserving normalizers includes all lexicographic algebras and all $\mathbb{Z}$-analytic algebras. We can describe many of the epimorphisms for these two classes of algebras, by using the results of the second two authors on primitivity [9].

We start with the lexicographic algebras and recall their construction from [24]. Let $(\Omega, \leq)$ be a countable linear ordering, i.e., let $\Omega$ be a countable set and $\leq$ a linear order on $\Omega$. Let $\omega: \mathbb{N} \rightarrow \Omega$ be an enumeration of $\Omega$ and let $\nu: \Omega \rightarrow$ $\{2,3,4, \ldots\}$ be a multiplicity function; set $\omega_{k}=\omega(k)$ and $\nu_{k}=\nu\left(\omega_{k}\right)$. Also let $F_{n}=\left\{\omega_{1}, \omega_{2}, \ldots, \omega_{n}\right\}$, for $n \in \mathbb{N}$. View $\mathbf{T}_{\nu_{1} \nu_{2} \ldots \nu_{n}}$ as the subalgebra $\mathcal{A}_{n}$ of $\mathbf{M}_{\nu_{1} \nu_{2} \ldots \nu_{n}}$ which is spanned by the matrix units

$$
e_{i j}=e_{\left(i_{1}, i_{2}, \ldots, i_{n}\right),\left(j_{1}, j_{2}, \ldots, j_{n}\right)},
$$

where $i \leq j$ if and only if there is some $k_{0} \in\{1,2, \ldots n\}$, depending on $i$ and $j$, such that $i_{k_{0}}<j_{k_{0}}$ and $i_{k}=j_{k}$ for all $\omega_{k}<\omega_{k_{0}}$ (notice that the indexing here is different than that of Section 2). Viewing $\mathcal{A}_{n+1}=\mathbf{T}_{\nu_{1} \nu_{2} \ldots \nu_{n+1}}$ in a similar manner, we define the map $\psi_{n}: \mathcal{A}_{n} \rightarrow \mathcal{A}_{n+1}$ to be the linear extension of the map which takes

$$
e_{i j} \mapsto \sum_{\xi=1}^{\nu_{n+1}} e_{\left(i_{1}, i_{2}, \ldots, i_{n}, \xi\right),\left(j_{1}, j_{2}, \ldots, j_{n}, \xi\right)} .
$$

It is clear that each $\psi_{n}$ is a $*$-extendible, regular embedding, and so the direct limit defines a triangular UHF algebra $\lim \left(\mathcal{A}_{n}, \psi_{n}\right)$, which we denote $A(\Omega, \nu)$. A standard argument using intertwining diagrams shows that a different enumeration of $\Omega$ will produce an isometrically isomorphic algebra, so $A(\Omega, \nu)$ is well-defined.

The algebras $A(\Omega, \nu)$, the lexicographic algebras, were studied in [24, [25] and were extended to subalgebras of direct sums of $\mathbf{M}_{n}$ 's in [14. The familiar standard, refinement, and alternation limit algebras are all examples of lexicographic algebras. Indeed, $\Omega=\mathbb{Z}_{-}, \mathbb{Z}_{+}$, and $\mathbb{Z}$ yields the standard, refinement, and alternation algebras, respectively.

Assume that $\Omega=\left\{\omega_{k} \mid k \in \Omega_{0}\right\}$ is ordered like a subset of the integers, i.e., $\Omega_{0} \subseteq \mathbb{Z} \backslash\{0\}$ and $\omega_{k} \leq \omega_{l}$ if $k \leq l$. Let $\nu: \Omega \rightarrow\{2,3,4, \ldots\}$ be as above. We write

$$
r_{k}=\nu\left(\omega_{k}\right) \quad \text { and } \quad s_{k}=\nu\left(\omega_{-k}\right), \quad k=1,2,3, \ldots
$$

with the understanding that if $k \notin \Omega_{0}$, then $r_{k}=1$, and, similarly, if $-k \notin \Omega_{0}$, then $s_{k}=1$. Associate with $\mu$ the pair $(\underline{r}, \underline{s})$ of (possibly) generalized integers

$$
\underline{r}=r_{1} r_{2} r_{3} \ldots \quad \text { and } \quad \underline{s}=s_{1} s_{2} s_{3} \ldots
$$

Define an equivalence relation $\sim$ on pairs of generalized (or finite) integers by $(\underline{r}, \underline{s}) \sim\left(\underline{r}^{\prime}, \underline{s}^{\prime}\right)$ if and only if $\underline{r s}=\underline{r}^{\prime} \underline{s}^{\prime}$ and there exist coprime natural numbers $a, b$ so that $b \underline{r}=a \underline{r}^{\prime}$ and $a \underline{s}=b \underline{s}^{\prime}$.

Assume now that $\Omega$ is an arbitrary countable linear ordering. Define an equivalence relation $\approx$ on $\Omega$ such that $\omega \approx \omega^{\prime}$ if the order intervals $\left[\omega, \omega^{\prime}\right]$ and $\left[\omega^{\prime}, \omega\right]$ are finite. Then the set $\Omega / \approx$ of equivalence classes is linearly ordered and each equivalence class $\langle\omega\rangle$ is itself a linearly ordered set isomorphic to a subset of the integers. Then to each equivalence class $\langle\omega\rangle$ we can associate a pair of generalized integers $p_{\nu}(\langle\omega\rangle)=(\underline{r}, \underline{s})$. 
Power's classification of lexicographic algebras [25, Theorems 4 and 6], after invoking Theorem [2.5, becomes:

Theorem 3.1. Let $\Omega, \Omega^{\prime}$ be countable linear orderings with maps $\nu: \omega \rightarrow\{2,3$, $4, \ldots\}$ and $\nu^{\prime}: \omega^{\prime} \rightarrow\{2,3,4, \ldots\}$.

Then $A(\Omega, \nu)$ and $A\left(\Omega^{\prime}, \nu^{\prime}\right)$ are isomorphic as complex algebras if and only if there is an order bijection $s: \Omega / \approx \rightarrow \Omega^{\prime} / \approx$ so that $p_{\nu^{\prime}}(s(\langle\omega\rangle))=p_{\nu}(\langle\omega\rangle)$.

By 24], the semisimple lexicographic algebras are precisely those $A\left(\Omega^{\prime}, \nu^{\prime}\right)$ where $\Omega^{\prime}$ has no minimal element. Further, by [9, Proposition 3.1], these algebras are all primitive, so this class is precisely the primitive lexicographic algebras.

Theorem 3.2. There is an epimorphism from $A(\Omega, \nu)$ onto $B$, a primitive Banach algebra, if and only if there is an order interval decomposition $\Omega=\widehat{\Omega} \oplus \widehat{\Omega}^{c}$, where $\Omega^{c}$ contains no minimal element, so that $A\left(\widehat{\Omega}^{c}, \nu \widehat{\Omega}^{c}\right)$ is isomorphic to $B$, as complex algebras.

In particular, if $B=A\left(\Omega^{\prime}, \nu^{\prime}\right)$ where, necessarily, $\Omega^{\prime}$ has no minimal element, then there is an order bijection $s: \widehat{\Omega}^{c} / \approx \rightarrow \Omega^{\prime} / \approx$ so that $p_{\nu^{\prime}}(s(\langle\omega\rangle))=p_{\nu}(\langle\omega\rangle)$, where $\omega \in \widehat{\Omega}^{c}$.

Proof. If $\varphi: A(\Omega, \nu) \rightarrow B$ is an epimorphism, then $\operatorname{ker} \varphi \subseteq A(\Omega, \nu)$ is a primitive ideal. Then by 9 , Theorem 3.6], there exists an order interval decomposition $\Omega=\widehat{\Omega} \oplus \widehat{\Omega}^{\mathrm{c}}$, where $\widehat{\Omega}^{\mathrm{c}}$ has no minimal element, so that, as a complex algebra, $B$ is isomorphic to

$$
A(\Omega, \nu) / \operatorname{ker} \varphi \approx A\left(\widehat{\Omega}^{\mathrm{c}},\left.\nu\right|_{\widehat{\Omega}^{\mathrm{c}}}\right) .
$$

If $B=A\left(\Omega^{\prime}, \nu^{\prime}\right)$, then the conclusion follows from Theorem 3.1 .

Theorem 3.2 is false if $B$ is not primitive. Indeed, it is easy to see that there is an epimorphism from the $2^{\infty}$ refinement algebra onto the $3 \cdot 2^{\infty}$ refinement.

Finally, we investigate the epimorphisms of $\mathbb{Z}$-analytic algebras. The most convenient definition of $\mathbb{Z}$-analyticity involves a cocycle on the spectrum, so we define cocycles precisely.

Recall the definition of spectrum from just before Theorem 2.3. Let $\mathcal{B}$ be the UHF $\mathrm{C}^{*}$-algebra corresponding to $\mathcal{A}$, and let $\mathcal{G}$ be the equivalence relation generated by $\mathrm{R}(\mathcal{A})$. A continuous function $c: \mathcal{G} \rightarrow \mathbb{R}$ is called a cocycle if $c(x, y)+c(y, z)=$ $c(x, z)$ for all points $(x, y),(y, z) \in \mathcal{G}$. The algebra $\mathcal{A}$ is analytic if there is a cocycle $c$ on $\mathcal{G}$ so that $c^{-1}([0, \infty))=\mathrm{R}(A)$. The standard, refinement, and alternation limit algebras are analytic [32, Examples 6.1-6.3]; for strongly maximal non-analytic algebras, see [13, Section 4], 23. Proposition 10.18], or [31, Example 3.2]. Algebras that are analytic by an integer-valued cocycle are called $\mathbb{Z}$-analytic [16, 18]. Although standard embedding algebras are the generic examples of $\mathbb{Z}$-analytic algebras, the paper [16] gives an example, due to Donsig and Hopenwasser, of a semisimple, $\mathbb{Z}$-analytic algebra which is not built up out of standard embeddings.

Theorem 3.3. Suppose $\mathcal{A}$ and $\mathcal{B}$ are $T A F$ algebras which are strongly maximal triangular in factors and that $\mathcal{A}$ is $\mathbb{Z}$-analytic. If $\varphi: \mathcal{A} \rightarrow \mathcal{B}$ is an epimorphism, then $\varphi$ is an isomorphism.

Proof. Since $\mathcal{B}$ is strongly maximal triangular in factors, the zero ideal of $\mathcal{B}$ is meet irreducible. By automatic continuity, the closed ideals of $\mathcal{A}$ containing $\operatorname{ker} \varphi$ form a sublattice of the closed ideals of $\mathcal{B}$. Hence $\operatorname{ker} \varphi$ is meet irreducible in $\mathcal{A}$. But $\mathcal{A}$ 
is $\mathbb{Z}$-analytic, so by [4, Proposition 4.6], $\operatorname{ker} \varphi$ is either zero or it must have finite codimension in $\mathfrak{A}$. But if $\operatorname{ker} \varphi$ had finite codimension, since $\mathcal{A} / \operatorname{ker} \varphi \simeq \mathcal{B}$, we would have that $\mathcal{B}$ is finite dimensional, a contradiction. Hence $\operatorname{ker} \varphi=(0)$, and so $\varphi$ is an isomorphism.

\section{REFERENCES}

1. R.L. Baker, Triangular UHF algebras, J. Funct. Anal. 91 (1990), 182-212. MR 91d:46056

2. H.G. Dales, R.J. Loy, and G.A. Willis, Homomorphisms and derivations from $B(E)$, J. Funct. Anal. 120 (1994), 201-219. MR 95b:46068

3. A. Donsig and A. Hopenwasser, Order preservation in limit algebras, J. Funct. Anal. 133 (1995), 342-394. MR 96k:46099

4. A. Donsig, A. Hopenwasser, T.D. Hudson, M.P. Lamoureux, and B. Solel, Meet irreducible ideals in direct limit algebras, Math. Scand. (to appear).

5. A. Donsig and T. Hudson, The lattice of ideals of a triangular AF algebra, J. Funct. Anal. 138 (1996), 1-39. MR 97e:47068

6. M. Eidelheit, On isomorphisms of rings of linear operators, Studia Math. 9 (1940), 97-105. MR 3:51e; MR 2:224d

7. A. Hopenwasser and S.C. Power, Classification of limits of triangular matrix algebras, Proc. Edinburgh Math. Soc. 36 (1992), 107-121. MR 94k:47068

8. T.D. Hudson, Ideals in triangular AF algebras, Proc. London Math. Soc. (3) 69 (1994), 345376. MR 95d:46060

9. T.D. Hudson and E.G. Katsoulis, Primitive triangular UHF algebras, J. Funct. Anal. 160 (1998) 1-27. MR 99m:46135

10. B.E. Johnson, Continuity of homomorphisms of algebras of operators, J. London Math. Soc. (2) 42 (1967), 537-541. MR 35:5953

11. 537-539. MR 35:2142

12. R.V. Kadison and J.R. Ringrose, Fundamentals of the theory of operator algebras, vol. I, Pure and Applied Mathematics, no. 100, Academic Press, San Diego, CA, 1983. MR 85j:46099

13. L. Mastrangelo, P.S. Muhly, and B. Solel, Locating the radical of a triangular operator algebra, Math. Proc. Cambridge Philos. Soc. 115 (1994), 27-38. MR 94m:46089

14. J.R. Peters and Y.T. Poon, Lexicographic TAF algebras, Trans. Amer. Math. Soc. 349 (1997), 4825-4855. MR 98d:47098

15. J.R. Peters, Y.T. Poon, and B.H. Wagner, Triangular AF algebras, J. Operator Theory 23 (1990), 81-114. MR 91h:46102

16. _ Analytic TAF algebras, Canad. J. Math. 45 (1993), 1009-1031; Correction to: Analytic TAF algebras, 46 (1994), 395-396. MR 94m:46113a; MR 94m:46113b

17. Y.T. Poon, A complete isomorphism invariant for a class of triangular UHF algebras, J. Operator Theory 27 (1992), 221-230. MR 95c:46093

18. Y.T. Poon and B.H. Wagner, Z-analytic TAF algebras and dynamical systems, Houston J. Math. 19 (1993), 181-199. MR 95f:46113

19. S.C. Power, On Ideals of Nest Subalgebras of $C^{*}$-algebras, Proc. London Math. Soc. (3) 50 (1985), 314-332. MR 86d:47057

20. Classification of tensor products of triangular operator algebras, Proc. London Math. Soc. (3) 61 (1990), 571-614. MR 92a:47053

21. - The classification of triangular subalgebras of $A F C^{*}$-algebras, Bull. London Math. Soc. 22 (1990), 269-272. MR 91e:46078

22. $ـ$ Algebraic order on $K_{0}$ and approximately finite operator algebras, J. Operator Theory 27 (1992), 87-106. MR 95b:46100

23. Limit algebras: an introduction to subalgebras of $C^{*}$-algebras, Pitman Research Notes in Mathematics, no. 278, Longman Scientific and Technical, London, 1992. MR 94g:46001

24. _ Infinite lexicographic products of triangular algebras, Bull. London Math. Soc. 27 (1995), 273-277. MR 96e:47047

25. _ Lexicographic semigroupoids, Ergodic Theory Dynamical Systems 16 (1996), 365377. MR 97d:47050

26. _ Errata for Limit algebras, available from http://www.maths.lancs.ac.uk/ power/

27. C. Qiu, Algebra isomorphisms of triangular operator algebras, preprint, 1993. 
28. C.E. Rickart, The uniqueness of norm problem in Banach algebras, Ann. of Math (2) 51 (1950), 615-628. MR 11:670d

29. G.E. Silov, On regular normed rings, Trav. Inst. Math. Stekloff 21 (1947), 118 pp. MR 9:596a

30. A.M. Sinclair, Homomorphisms from $C_{0}(R)$, J. London Math. Soc. (2) 11 (1975), 165-174. MR 51:13689

31. B. Solel and B. Ventura, Analyticity in triangular UHF algebras, J. Operator Theory 28 (1992), 357-370. MR 95i:46092

32. B. Ventura, Strongly maximal triangular AF algebras, Internat. J. Math. 2 (1991), 567-598. MR 92k:46096

Department of Mathematics and Statistics, University of Nebraska at Lincoln, LinCOLN, NeBRAska 68588-0323

E-mail address: adonsig@math.unl.edu

Department of Mathematics, East Carolina University, Greenville, North Carolina $27858-4353$

E-mail address: tdh@math.ecu.edu

Department of Mathematics, East Carolina University, Greenville, North Carolina $27858-4353$

E-mail address: katsoulise@mail.ecu.edu 